\title{
Quantifying particle dispersal in aquatic sediments at short time scales: model selection
}

\author{
Filip J. R. Meysman ${ }^{1,2, *}$, Volodymyr S. Malyuga1 ${ }^{1}$, Bernard P. Boudreau ${ }^{3}$, \\ Jack J. Middelburg ${ }^{1}$
}

${ }^{1}$ The Netherlands Institute of Ecology (NIOO-KNAW), Centre for Estuarine and Marine Ecology, PO Box 140, 4400 AC Yerseke, The Netherlands

${ }^{2}$ Department of Analytical and Environmental Chemistry, Vrije Universiteit Brussel (VUB), Pleinlaan 2, 1050 Brussel, Belgium ${ }^{3}$ Department of Oceanography, Dalhousie University, Halifax, Nova Scotia B3H 4J1, Canada

\begin{abstract}
In a pulse-tracer experiment, a layer of tracer particles is added to the sediment-water interface, and the down-mixing of these particles is followed over a short time scale. Here, we compared different models (biodiffusion, telegraph, CTRW) to analyse the resulting tracer depth profiles. The biodiffusion model is widely applied, but entails 2 problems: (1) infinite propagation speed - the infinitely fast propagation of tracer to depth, and (2) infinitely short waiting times - mixing events follow each other infinitely fast. We show that the problem of waiting times is far more relevant to tracer studies than the problem of propagation speed. The key issue in pulse-tracer experiments is that models should explicitly account for a finite waiting time between mixing events. The telegraph equation has a finite propagation speed, but it still assumes infinitely short waiting times, and, hence, it does not form a suitable alternative to the biodiffusion model. Therefore, we advance the continuous-time random walk (CTRW), which explicitly accounts for finite waiting times between mixing events, as a suitable description of bioturbation. CTRW models are able to cope with lateral spatial heterogeneity in reworking, which is a crucial feature of bioturbation at short time scales. We show how existing bioturbation models (biodiffusion model, telegraph equation, non-local exchange model) can be considered as special cases of the CTRW model. Accordingly, the CTRW model is not a new bioturbation model, but a generalization of existing models.
\end{abstract}

KEY WORDS: Bioturbation · Diffusion $\cdot$ Luminophores $\cdot$ Modelling $\cdot$ Continuous-time random walk

\section{INTRODUCTION}

Aquatic sediments are continuously reworked due to the activity of local infauna, a process typically referred to as bioturbation (Richter 1952). Bioturbation results from a wide range of animal behaviours, such as burrow and tube excavation (including the ultimate collapse and refilling of these tubes), feeding and defecation, crawling and ploughing through the sediment, the building of mounds and the digging of craters (Rhoads 1974, Cadée 2001, Solan \& Wigham 2005). Bioturbation also forms the driving force for the dispersal of various solid particles, which can be both mineral (e.g. organic matter, metal oxides and contaminants) as well as biological (e.g. bacteria, viruses, cysts and resting stages of plankton) in nature. As a consequence, bioturbation plays a crucial role in the geochemistry and ecology of aquatic sediments (Aller \& Yingst 1978, Aller 1988, Meysman et al. 2006). To further our understanding of these subsurface environments, it is of prime interest to quantitatively describe the particle dispersal induced by bioturbation.

To date, the most commonly employed bioturbation model is the biodiffusion analogy, which assumes that Fick's laws of diffusion are applicable to macrofaunainduced particle dispersal (Goldberg \& Koide 1962, Guinasso \& Schink 1975, Boudreau 1986). By fitting suitable solutions of the biodiffusion model to tracer 
depth profiles, the mixing intensity is quantified by a single parameter, the diffusion coefficient $D_{\mathrm{b}}$. In recent years, the validity and accuracy of the biodiffusion model has, however, been questioned for different reasons (Boudreau 1989, Meysman et al. 2003, Reed et al. 2006). A first issue was brought up by Boudreau (1989), who noted that the biodiffusion model inherently has an infinite speed of signal propagation. Immediately after releasing a tracer pulse, the biodiffusion model will predict that the tracer is already present far away from the source, which is physically unrealistic. This means that the biodiffusion model will generate biased predictions at short time spans. Okubo (1971) illustrated this problem for oceanic turbulence: if one releases $2 \mathrm{~kg}$ of salt off the coast of California, a diffusive model of turbulence would predict to find 1 molecule in $500 \mathrm{l}$ of water off the coast of Japan in 15 mo. This molecule then would have travelled with a mean velocity of $25 \mathrm{~cm} \mathrm{~s}^{-1}$, which is highly unrealistic. Accordingly, to describe natural mixing processes at short time scales, models with a finite signal propagation velocity are preferential from a theoretical point of view. In this light, Boudreau (1989) suggested that the telegraph equation (see below Eq. 33) could be an alternative to the diffusion equation. The telegraph equation has indeed a finite velocity of signal propagation (Monin 1959, Okubo 1971), and, hence, it would resolve the problem of unphysical fast penetration of tracer at depth. Yet up to present, the telegraph equation has not been applied in bioturbation studies.

A second objection to the biodiffusion model was advanced by Meysman et al. (2003): its assumptions are violated at short time scales. But how 'short' is a short time scale? Based on a theoretical analysis of random-walk models, it was argued that the biodiffusion model requires that the inherent time scale of the mixing process should be considerably smaller than the observational time scale. The inherent time scale of the mixing process then refers to the average time interval between 2 displacements of a particle. In contrast, the observational time scale denotes the period over which bioturbation is studied. In other words, Meysman et al. (2003) argued that the biodiffusion model can only be used when the observational period is long enough so that it 'captures' a sufficiently large number of mixing events. This 'frequency' constraint is illustrated by the following hypothetical example. Suppose that bioturbation is studied at a given site with the common radiotracer methods ${ }^{234} \mathrm{Th}$ and ${ }^{210} \mathrm{~Pb}$, which have respective half-lives of $24.1 \mathrm{~d}$ and $22.3 \mathrm{yr}$. The observational time scale is proportional to the half-life of the tracer, because the characteristic period over which radiotracer-coated particles still can be followed is about 5 times the half-life. Constraining the time scale of the mixing is more difficult, because at present we do not have accurate estimates of the time interval between displacements in natural environments (this is one of the major challenges in bioturbation research-see Maire et al. 2007). But let us assume that particles are moved once every day on average. Accordingly, the 'captured' number of particle displacement events greatly differs between the 2 methods: 8140 events for ${ }^{210} \mathrm{~Pb}$, but only 24 events for ${ }^{234} \mathrm{Th}$. Accordingly, compared to the mixing time scale of $1 \mathrm{~d},{ }^{234} \mathrm{Th}$ is qualified as a short time scale method, while the ${ }^{210} \mathrm{~Pb}$ method classifies as a long time scale method. This emphasizes the relative nature of the term 'short': short means short with respect to the inherent time scale of the mixing, which may drastically vary between environments (e.g. deep sea versus coastal sediments).

So why would the biodiffusion model in the example above be more suited to analyse the ${ }^{210} \mathrm{~Pb}$ activity profile as opposed to the ${ }^{234} \mathrm{Th}$ profile? The prime reason is that the biodiffusion model implicitly assumes that mixing events follow each other infinitely fast, so that infinitely many mixing events are thought to take place within the observational period. When particle dispersal is observed over sufficiently long time scales (e.g. with long-lived radiotracers such as ${ }^{210} \mathrm{~Pb}$ ), the time between 2 mixing events is small compared to the period of observation, and so the idealization of infinitely frequent mixing may be justified. However, when particle dispersal is observed over shorter time scales (e.g. with short-lived radiotracers such as ${ }^{234} \mathrm{Th}$ ), the observed number of mixing events is reduced, and so the biodiffusion model may no longer be applicable. Recently, Reed et al. (2006) convincingly demonstrated this breakdown of the biodiffusion model at short time scales using a lattice-automaton model environment (this is basically a virtual sediment environment in which bioturbation experiments can be simulated). These simulations revealed that biodiffusion coefficients estimated from steady-state profiles of shortlived isotopes showed a conspicuous tracer dependence. Overall, biodiffusion coefficients based on short-lived isotopes were strongly biased towards larger values and had large standard deviations.

As in the case of methods based on steady-state profiles of short-lived isotopes, pulse-tracer experiments typically have short observation windows. Because the time scale of experiments is tuned to the expected time scale of mixing, observation times range from $1 \mathrm{~d}$ in coastal areas (e.g. Fornes et al. 1999, Solan et al. 2004) over 1 mo in laboratory microcosms (e.g. Fernandes et al. 2006) to $1 \mathrm{yr}$ in the deep sea (Wheatcroft 1991). In the pulse-tracer method, a layer of tracer particles is deposited onto the sediment-water interface (SWI), and its subsequent down-mixing into the sediment is followed. Typical tracer particles are inert particles, such as glass beads (e.g. Shull \& Yasuda 2001) and 
fluorescent luminophores (Mahaut \& Graf 1987), or particles tagged with short-lived radionuclides (Fornes et al. 1999, 2001). The final output of a tracer-pulse experiment is a set of tracer-depth profiles, collected at different time intervals. Our principal aim here is to examine what model is most appropriate for analyzing such tracer profiles. Is the biodiffusion model applicable, or should we search for alternative model formulations?

To examine this, we compared the performance of the biodiffusion model to 2 alternative models: (1) the telegraph equation, which was proposed by Boudreau (1989) on theoretical grounds, but the applicability of which as a bioturbation model has not been examined yet; (2) the continuous-time random-walk (CTRW) model, which has recently received substantial attention in the field of statistical physics (Metzler \& Klafter 2000). We examined the CTRW model as a new alternative to the biodiffusion model. The CTRW bioturbation model, which is presented in detail here, has been applied by Maire et al. (2007) to analyse luminophore data obtained via a high-resolution imaging technique. This work revealed that the CTRW model provided better and more robust fits to the data than the biodiffusion model, particularly at short time scales. Here, our main ambition is to provide a theoretical framework for these results: Why does the CTRW perform better at short time scales and how does it relate to classical bioturbation models such as the biodiffusion and non-local exchange models? In a first step, we detail the model formulation and numerical solution of the CTRW model. In a second step, we show how the biodiffusion and telegraph models can be obtained as limiting cases of the CTRW model, and how the assumptions of an infinite signal propagation speed and infinite mixing frequency are linked to these limiting conditions. In a final step, we perform simulations of a pulse-tracer experiment and compare the simulation output of the biodiffusion, telegraph and general CTRW models.

\section{MODEL FORMULATION}

\section{Modelling approach}

Many different organisms are present in the sediment, and each organism displays a range of different particle displacement activities, thus providing an insurmountable number of possibilities for the movement of a given particle. Because of this complexity, we cannot deterministically describe the motion of each single particle in the sediment. A convenient solution to this problem is to assume that the interplay between particles and biological activity is sufficiently erratic, so that a particle's motion can be described as a random process (Boudreau 1986, Wheatcroft et al. 1990, Meysman et al. 2003). Adopting a stochastic perspective, particle dispersal due to bioturbation can be regarded as a random walk, which is essentially a mathematical formalization of the intuitive idea of taking successive steps, each in a random direction (Hughes 1995). The consecutive movement of a bioturbated particle is principally governed by 3 quantities: (1) the jump direction, (2) the jump distance and (3) the waiting time between jumps or bioturbation events. Each of these quantities can be modelled in either a deterministic or a stochastic way, and, depending on these choices, one will obtain different random-walk models. When all variables (jump direction, jump distance and waiting time) are true stochastic variables, each modelled by a suitable probability distribution, the resulting description of particle dispersal is referred to as a continuous-time random walk (CTRW).

\section{General CTRW formulation}

From a stochastic perspective, one can regard particle displacement as a random sequence of bioturbation events, e.g. the infilling of a burrow or the passage of a crawling organism. In this view, a bioturbated particle displays 2 types of 'behaviour': (1) 'jumping' to a new location during a given bioturbation event and (2) 'waiting' at a given location until the next bioturbation event occurs (Fig. 1). When casting this into a mathematical model, 2 basic parameters need specification: (1) the jump vector $\mathbf{J}$, i.e. the direction and distance a particle travels in a given jump, and (2) the waiting time $T$, i.e. the time a particle waits between 2 jumps. In the analogy of the wandering drunkard, the vector $\mathbf{J}$

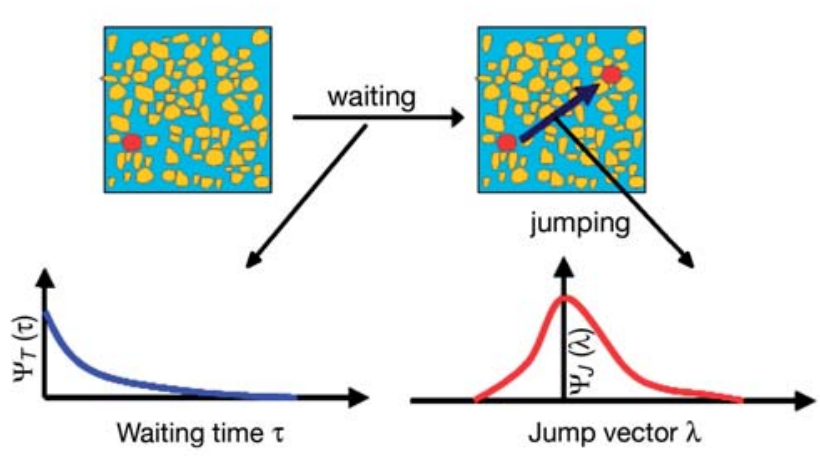

Fig. 1. Idealization of particle displacement as a position jump process. Particles display 2 behavioural modes: long waits (waiting time $T$ ) and fast jumps (jump vector $\mathbf{J}$, which in 1 dimension becomes the jump length $\lambda$ ). In a continuous-time random-walk model, these 2 quantities are described by a probability distribution function termed the waiting time and jump vector distribution, respectively 
models the actual steps that are taken, while $T$ represents the time the drunkard hesitates between steps. The CTRW model (Montroll \& Weiss 1965, Hughes 1995, Metzler \& Klafter 2000) assumes that both the jump vector $\mathbf{J}$ and the waiting time $T$ are drawn from a joint probability distribution function (PDF). When a particle is located at point $\mathbf{x}^{\prime}$ at time $t^{\prime}$, the probability of finding this particle at the new location $\mathbf{x}$ at a later time $t$ is given by the so-called jump distribution (Metzler \& Klafter 2000) such that:

$$
\begin{aligned}
\Psi\left(\mathbf{x}, t_{;} \mathbf{x}^{\prime}, t^{\prime}\right) \mathrm{d} t \mathrm{~d} \mathbf{x}= & \operatorname{Pr}\left\{t-t^{\prime}<T<t+\mathrm{d} t-t^{\prime} ;\right. \\
& \left.\mathbf{x}-\mathbf{x}^{\prime}<\mathbf{J}<\mathbf{x}+\mathrm{d} \mathbf{x}-\mathbf{x}^{\prime}\right\}
\end{aligned}
$$

The notation $\operatorname{Pr}\{\ldots\}$ refers to the probability that a certain 'event' takes place (see general texts on stochastic processes and multivariate distributions, e.g. Gardiner 2002). The actual form of the jump vector $\mathbf{J}$ will depend on the dimensionality of the problem and the coordinate system in use. In the general 3-dimensional case, the position jump model will incorporate 4 degrees of freedom, i.e. 3 spatial (the 3 components of J) and 1 temporal $(T)$. In 3D Cartesian coordinates, the jump vector is then given by $\mathbf{J}=\mathbf{J}\left(L_{x}, L_{y}, L_{z}\right)$, where $L_{x}$, $L_{y}$ and $L_{z}$ denote the jump lengths along the $x^{-}, y^{-}$and $z$-axis, respectively, each having a range $-\infty \leq L \leq+\infty$.

\section{Simplifying assumptions}

The idea is now to develop the general CTRW description (Eq. 1) into an actual bioturbation model. In doing so, we adopt some suitable simplifications (see Meysman et al. 2008) for an in-depth discussion of these idealizations. Firstly, the probability distribution $\Psi$ remains invariant with time (temporal homogeneity) and does not vary over the spatial domain (spatial homogeneity). Secondly, the jump vector $\mathbf{J}$ and the waiting time $T$ are considered independent variables, and, as a result, the joint probability distribution $\Psi$ can be decomposed into 2 separate units such that:

$$
\Psi\left(\mathbf{x}_{1} t_{;} \mathbf{x}^{\prime}, t^{\prime}\right) \mathrm{d} t \mathrm{~d} \mathbf{x}=\Psi_{T}\left(t_{;} t^{\prime}\right) \Psi_{\mathbf{J}}\left(\mathbf{x}_{;} \mathbf{x}^{\prime}\right) \mathrm{d} t \mathrm{~d} \mathbf{x}
$$

where $\Psi_{\mathbf{J}}$ is referred to as the jump vector distribution and $\Psi_{T}$ is the waiting time distribution. Thirdly, our bioturbation models are restricted to 1 dimension, a constraint that results from the type of data that are normally available (tracer depth profiles). We use a single coordinate $x$ that represents the depth into the sediment. As particles can only travel in 1 dimension, the jump vector $\mathbf{J}$ is represented in the Euclidean form $\mathbf{J}(L, 0,0)$, where the scalar quantity $L$ is usually referred to as the jump length (Metzler \& Klafter 2000). Note that the jump length $L$ can be either positive or negative, and, hence, it also accounts for the direction of particle movement. The value of $L$ is positive when the direction of the jump coincides with the direction of the $x$-axis (particles move downwards into the sediment) and is negative otherwise (particles move upwards). When a given particle is located at point $x^{\prime}$ at time $t^{\prime}$, the probability of finding a particle within the interval $(x, x+\mathrm{d} x)$ at some time from $(t, t+\mathrm{d} t)$ can be written as:

$$
\begin{aligned}
\Psi\left(x, t_{;} x^{\prime}, t^{\prime}\right) \mathrm{d} t \mathrm{~d} x & =\Psi_{T}\left(t-t^{\prime}\right) \Psi_{L}\left(x-x^{\prime}\right) \mathrm{d} t \mathrm{~d} x \\
& =\Psi_{T}(\tau) \Psi_{L}(\lambda) \mathrm{d} \tau \mathrm{d} \lambda
\end{aligned}
$$

Eq. (3) is the implementation of the general model (Eq. 2) in 1 dimension. The first factor on the right is again the waiting time distribution with $\tau \equiv t-t^{\prime}$. The second factor is the jump length distribution with $\lambda \equiv x-x^{\prime}$. Both the waiting time and jump length distributions are now of the convolutive form. Note that Eq. (3) still allows for drift. This can occur when the jump length distribution is non-symmetric, i.e. $\Psi_{L}(-\lambda) \neq \Psi_{L}(\lambda)$, although here we will mainly consider symmetric distributions.

\section{Governing equations}

The jump distribution (Eq. 3) describes the effect of a single bioturbation event on the location of a single particle. To quantify the effect of many bioturbation events on a single particle, we can release a certain particle at the origin and analyse the evolution of position. The probability $P(x, t \mid 0,0)$ of finding such a particle at depth $x$ after some time $t$ is given (Othmer et al. 1988, Meysman et al. 2008) as:

$$
\begin{aligned}
P(x, t \mid 0,0)= & \delta(x)\left[1-\int_{0}^{t} \Psi_{T}(\tau) \mathrm{d} \tau\right] \\
& +\int_{0}^{t} \int_{-\infty}^{\infty} \Psi_{T}(\tau) \Psi_{L}(\lambda) P(x-\lambda, t-\tau \mid 0,0) \mathrm{d} \lambda \mathrm{d} \tau
\end{aligned}
$$

where $\delta(x)$ is the Dirac delta function. Eq. (4) is termed a renewal equation, and describes the stochastic behaviour of a single particle after many bioturbation events (Othmer et al. 1988). The first term on the righthand side of Eq. (4) expresses the probability that a particle remains 'unmoved' at the origin. The second (integral) term accounts for the 'behaviour' of the particle when it effectively leaves its initial position. The renewal equation (Eq. 4) thus represents a stochastic model for the position of a single particle. However, the goal of any bioturbation model is to deterministically describe the effect of many bioturbation events on the location of many particles. To go from the 'stochastic/single particle' level to the 'deterministic/many particles' level, we can invoke the law of large numbers (Feller 1968). This law basically implies that the average of a random sample from a large population is likely to be close to the mean of the whole population. Invoking the law of large numbers, the concentration 
of particles at an arbitrary location and time can be expressed as:

$$
\begin{aligned}
C(x, t)= & C_{0}(x)\left[1-\int_{0}^{t} \Psi_{T}(\tau) \mathrm{d} \tau\right] \\
& +\int_{0}^{t} \int_{-\infty}^{\infty} \Psi_{T}(\tau) \Psi_{L}(\lambda) C(x-\lambda, t-\tau) \mathrm{d} \lambda \mathrm{d} \tau
\end{aligned}
$$

where the initial concentration distribution of particles is given by:

$$
C_{0}(x)=C(x, 0)
$$

The initial value problem as specified by Eqs. (5) \& (6) forms the complete statement of our CTRW model of bioturbation. Given a particular waiting time PDF $\Psi_{T}(\tau)$ and a step length PDF $\Psi_{L}(\lambda)$, Eq. (5) will predict how the initial tracer profile $C_{0}(x)$ will evolve with time. Together, the distributions $\Psi_{T}(\tau)$ and $\Psi_{L}(\lambda)$ may be regarded as the 'bioturbation fingerprint' for a given macrofaunal community. Obviously, this fingerprint will depend on the organisms that are present (species composition, size, abundance) and on the specific activities involved (e.g. deposit-feeding, burrowing).

\section{Reflection at the sediment-water interface (SWI)}

Until now, we have implicitly assumed that the jump distribution (Eq. 3) applies to the infinite domain $-\infty<x<\infty$, and we will refer to the associated jump length distribution as $\Psi_{L}^{\text {inf }}$. In reality, however, the sediment is bounded by the SWI, and so particle dispersal only occurs within the semi-infinite domain $0<x<\infty$. Here, we do not consider the removal of particles due to resuspension or erosion, nor the increase of particles due to sedimentation. So, any particle that is transported across the SWI must return to the bioturbated zone. Particles ejected from the sediment will settle in a random fashion at the SWI. A realistic description of this process would, however, drastically increase the mathematical complexity of the model. To avoid such complexity, we adopt the most parsimonious boundary condition: the SWI acts as a perfectly reflective barrier. Moreover, this idealization does not influence the discussion and conclusions arrived upon below. Mathematically, this reflection implies that the original jump length distribution $\Psi_{L}^{\text {inf }}$ is mirrored around the origin, and that the mirrored 'tail' is added to the original jump length distribution (Fig. 2). The probability that a particle starts at $x^{\prime}$ and arrives within the interval $(x, x+d x)$ is now composed of 2 parts: the chance that it moves to this interval directly, and the chance that it moves indirectly to $(x, x+d x)$ after reflection at the SWI. The latter probability is the same as that it would start at $X^{\prime}$ and arrive within the neighbourhood of $-X$ :

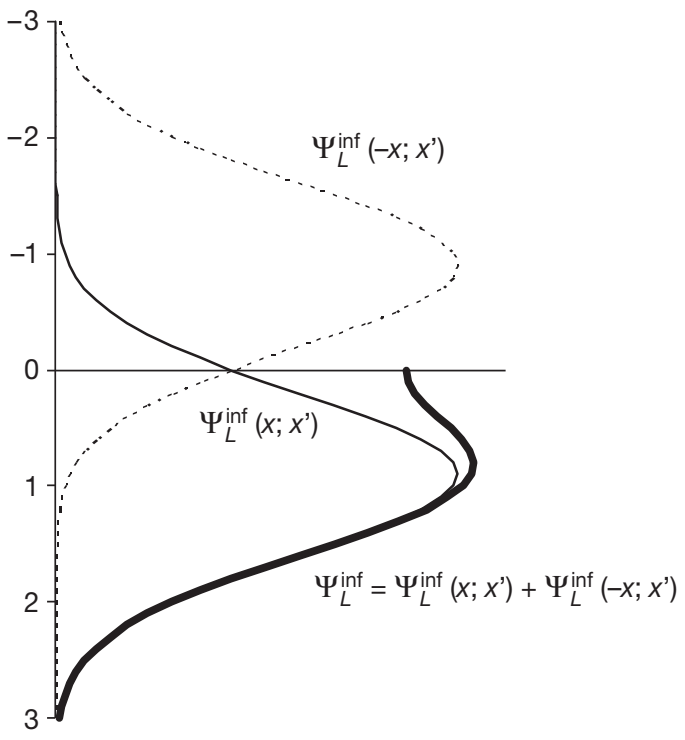

Fig. 2. Reflection of particles at the sediment-water interface. The jump length distribution consists of the sum of 2 components: one part accounts for direct travel, the other part accounts for reflected particles

$$
\Psi_{L}\left(x ; x^{\prime}\right)=\Psi_{L}^{\inf }\left(x ; x^{\prime}\right)+\Psi_{L}^{\inf }\left(-X ; X^{\prime}\right)
$$

Using the convolutive form of Eq. (3) and implementing the coordinate change $\lambda \equiv x-x^{\prime}$, we directly obtain:

$$
\Psi_{L}(\lambda)=\Psi_{L}^{\inf }(\lambda)+\Psi_{L}^{\inf }(-2 X+\lambda)
$$

This new jump length distribution $\Psi_{L}$ must only be implemented over the semi-infinite domain $0 \leq x<\infty$. Accordingly, the initial conditions (Eq. 6) only apply to the semi-infinite domain, and the upper boundary of integration in Eq. (5) should be adapted, so that the variable $\lambda$ starts at $-\infty$ and only runs to $x$ :

$$
\begin{aligned}
C(x, t)= & C_{0}(x)\left[1-\int_{0}^{t} \Psi_{T}(\tau) \mathrm{d} \tau\right]+\int_{0}^{t} \int_{-\infty}^{x} \Psi_{T}(\tau)\left[\Psi_{L}^{\inf }(\lambda)\right. \\
& \left.+\Psi_{L}^{\inf }(-2 x+\lambda)\right] C(x-\lambda, t-\tau) \mathrm{d} \lambda \mathrm{d} \tau
\end{aligned}
$$

This integral equation forms the statement of our CTRW model of bioturbation. In the general case, Eq. (9) should be solved numerically. However, under the special condition that the jump length time distribution $\Psi_{L}^{\text {inf }}$ is symmetrical (as we assume here), one can transform Eq. (9) into a form that allows a simplified numerical solution. To see this, we first introduce the coordinate $z=x-\lambda$, so that Eq. (9) becomes:

$$
\begin{aligned}
C(x, t)= & C_{0}(x)\left[1-\int_{0}^{t} \Psi_{T}(\tau) \mathrm{d} \tau\right] \\
& +\int_{0}^{t} \int_{0}^{\infty} \Psi_{T}(\tau) \Psi_{L}^{\inf }(x-z) C(z, t-\tau) \mathrm{d} z \mathrm{~d} \tau \\
& +\int_{0}^{t} \int_{0}^{\infty} \Psi_{T}(\tau) \Psi_{L}^{\inf }(-x-z) C(z, t-\tau) \mathrm{d} z \mathrm{~d} \tau
\end{aligned}
$$


Using the symmetry of the jump length distribution $\Psi_{L}^{\inf }(-X-Z)=\Psi_{L}^{\inf }(X+Z)$, and changing the integration variable from $z$ to $(-z)$ in the second integral, this expression is re-arranged to:

$$
\begin{aligned}
C(x, t)= & C_{0}(x)\left[1-\int_{0}^{t} \Psi_{T}(\tau) \mathrm{d} \tau\right] \\
& +\int_{0}^{t} \int_{0}^{\infty} \Psi_{T}(\tau) \Psi_{L}^{\inf }(x-z) C(z, t-\tau) \mathrm{d} z \mathrm{~d} \tau \\
& +\int_{0}^{t} \int_{-\infty}^{0} \Psi_{T}(\tau) \Psi_{L}^{\inf }(x-z) C(-z, t-\tau) \mathrm{d} z \mathrm{~d} \tau
\end{aligned}
$$

In Eq. (11), the concentration $C(x, t)$ is still only defined over the semi-infinite domain $0 \leq x<\infty$. We now introduce a new concentration $\bar{C}$ over the whole infinite domain:

$$
\bar{C}(x, t)= \begin{cases}C(x, t) & \text { when } x \geq 0 \\ C(-x, t) & \text { when } x<0\end{cases}
$$

Reversing the coordinate definition $z=x-\lambda$ from above, Eq. (11) can be rewritten as:

$$
\begin{aligned}
\bar{C}(x, t)= & \bar{C}_{0}(x)\left[1-\int_{0}^{t} \Psi_{T}(\tau) \mathrm{d} \tau\right] \\
& +\int_{0}^{t} \int_{-\infty}^{\infty} \Psi_{T}(\tau) \Psi_{L}^{\inf }(\tau) \bar{C}(x-\lambda, t-\tau) \mathrm{d} \lambda \mathrm{d} \tau
\end{aligned}
$$

Remarkably, the integral Eq. (13), stated in terms of $\bar{C}$, is identical to the original form (Eq. 5), stated in terms of $C$, which, however, did not account for the SWI. Nonetheless, the solution is not the same. The difference is that the original initial conditions $C_{0}$ (as defined in Eq. 6) should be mirrored around the origin to arrive at the new initial conditions $\bar{C}_{0}$ :

$$
\bar{C}_{0}(x)= \begin{cases}C_{0}(x) & \text { when } x \geq 0 \\ C_{0}(-x) & \text { when } x<0\end{cases}
$$

Accordingly, by a simple reflection of the initial conditions $C_{0}$ around the origin, we are able to explicitly account for the presence of the SWI. This is the way the CTRW problem is solved here. For notational simplicity, we will from now on drop the bar symbols above the concentrations, and drop the 'inf' superscript for the jump length distribution.

\section{MODEL SOLUTION}

The solution of the initial value problem (Eqs. 13 \& 14) is obtained via a semi-analytical solution procedure, which involves a Fourier transformation to the spatial coordinate and a Laplace transformation to the time coordinate. In the transformed plane, the problem is then solved analytically. To arrive at the final solution in the original $(x, t)$ plane, the resulting Fourier and Laplace integrals need to be evaluated numerically.

\section{Fourier and Laplace transforms}

In a first step, we can apply the Fourier transform to Eq. (13) with respect to spatial coordinates. Using the convolution theorem, we thus obtain:

$\hat{C}(k, t)=\hat{C}_{0}(k)\left[1-\int_{0}^{t} \Psi_{T}(\tau) \mathrm{d} \tau\right]+\int_{0}^{t} \Psi_{T}(\tau) \hat{\Psi}_{L}(k) \hat{C}(k, t-\tau) \mathrm{d} \tau$

where the hat notation is used to denote the Fourier transform. In a second step, we apply the Laplace transform to Eq. (15) with respect to time. Again using the convolution theorem, we arrive at:

$$
\hat{\tilde{C}}(k, s)=\frac{1}{s}\left[1-\tilde{\Psi}_{T}(s)\right] \hat{C}_{0}(k)+\tilde{\Psi}_{T}(s) \hat{\Psi}_{L}(k) \hat{\tilde{C}}(k, s)
$$

where the tilde notation denotes the Laplace transform. By solving the algebraic equation (Eq. 16), we directly obtain the transform of the unknown concentration profile as:

$$
\hat{\tilde{C}}(k, s)=\frac{1-\tilde{\Psi}_{T}(s)}{s\left[1-\tilde{\Psi}_{T}(s) \hat{\Psi}_{L}(k)\right]} \hat{C}_{0}(k)
$$

Upon back transformation of Eq. (17), the concentration profile thus becomes:

$$
\begin{aligned}
C(x, t)= & \frac{1}{4 \pi^{2} i} \int_{-\infty}^{\infty} \hat{C}_{0}(k) \exp (-i k x) \\
& \int_{\gamma-i \infty}^{\gamma+i \infty} \frac{1-\tilde{\Psi}_{T}(s)}{s\left[1-\tilde{\Psi}_{T}(s) \hat{\Psi}_{L}(k)\right]} \exp (s t) \mathrm{d} s \mathrm{~d} k
\end{aligned}
$$

where $\gamma$ is a real number so that the contour path of integration is in the region of convergence of the Laplace transform.

\section{Numerical evaluation of integrals}

Eq. (18) is not solved as such, but is first reformulated in terms of real integrals before numerical integration. This procedure is detailed in Appendix 1, and eventually leads to the integral equation (Eq. A3), which only contains integrals that consist of sums of real Fourier sine and cosine functions. For the evaluation of these integrals, we employed the DQDAWF routine from the IMSL Fortran 90 MP Library Version 4.01. This routine approximates the Fourier integrals by repeated calls to the IMSL routine DQDAWO, which integrates functions $f(x) \sin (\omega x)$ or $f(x) \cos (\omega x)$ over a finite interval, followed by extrapolation. Depending on the length of the subinterval in relation to the value of $\omega$, either a modified Clenshaw-Curtis procedure or a Gauss-Kronrod 7/15 rule is employed by the DQDAWO routine to approximate the integral on a subinterval. In addition, this routine uses the $\varepsilon$-algorithm for the extrapolation. The routines DQDAWF and DQDAWO are implementations of the subroutines QAWF and QAWO, respectively, fully documented by Piessens et al. (1983). 


\section{BENCHMARK SOLUTION: BIODIFFUSION}

In order to check the accuracy and consistency of the numerical solution procedure, it is useful to compare the numerical solution to a corresponding analytical model solution (model verification). To achieve this, we use a result from random-walk theory with regard to the long-time behaviour of the CTRW model (Eq. 5). Effectively, one can prove (Hughes 1995, Metzler \& Klafter 2000) that if the waiting time distribution $\Psi_{T}$ has a finite mean:

$$
\tau_{C}=\int_{0}^{\infty} \tau \Psi_{T}(\tau) \mathrm{d} \tau
$$

and the jump length distribution $\Psi_{L}$ is symmetrical, i.e. $\Psi_{L}(\lambda)=\Psi_{L}(-\lambda)$, and has a finite variance:

$$
\sigma^{2}=\int_{-\infty}^{\infty} \lambda^{2} \Psi_{L}(\lambda) \mathrm{d} \lambda
$$

then, for sufficiently long times, $t \gg \tau_{C}$, the solution $C(x, t)$ of the CTRW equation (Eq. 5) will approach the solution of the diffusion equation:

$$
\frac{\partial C}{\partial t}=\frac{\sigma^{2}}{2 \tau_{c}} \frac{\partial^{2} C}{\partial x^{2}}
$$

In other words, the long-time effect of bioturbation will always look like diffusive mixing irrespective of the actual bioturbation fingerprint $\Psi_{T}$ and $\Psi_{L}$ that characterizes the benthic community. The long-time diffusion equation (Eq. 21) provides the following interpretation of the biodiffusion coefficient:

$$
D_{\mathrm{b}}=\frac{\sigma^{2}}{2 \tau_{c}}
$$

The root mean square variance $\sigma$ of the jump length distribution $\Psi_{L}$ thus serves as a characteristic length scale of mixing (the average displacement of a particle in a bioturbation event), and mean $\tau_{c}$ of the waiting time distribution $\Psi_{T}$ thus serves as the characteristic time scale of mixing (the average time between 2 displacements of a particle). This scaling result is similar, but not entirely analogous to previous decompositions of the biodiffusion coefficient in Wheatcroft et al. (1990) and Meysman et al. (2003).

Effectively, the relation between the CTRW model and the biodiffusion model has many facets, and a detailed discussion of these is beyond our scope here (see Meysman et al. 2008). For the present purposes 2 points are important: (1) For each CTRW model, we can construct an associated biodiffusion model that has exactly the same mixing intensity; ultimately, at sufficiently long times, the solutions of the CTRW and biodiffusion models should converge. (2) We can take advantage of the 'diffusive' behaviour of the CTRW model at long times to verify the accuracy of our numerical solution procedure. To this end, we proceed as follows. We first calculate the mean waiting time
(Eq. 19) and the jump length variance (Eq. 20) for a given bioturbation fingerprint, i.e. a combination of distributions $\Psi_{T}$ and $\Psi_{L}$. Subsequently, we calculate the biodiffusion coefficient via Eq. (22), and we solve the diffusion equation (Eq. 21) for exactly the same initial conditions (Eq. 6). After a sufficiently long time $t \gg \tau_{C}$, the solution of the CTRW equation (Eq. 18) should match the solution of the 'diffusive' approximation (Eq. 21).

\section{CONNECTION TO EXISTING BIOTURBATION MODELS}

The novelty of the CTRW approach lies in the fact that the waiting time between bioturbation events is no longer fixed, but is instead governed by a probability distribution. Yet the CTRW equation (Eq. 5) is not a 'new' bioturbation model, but a generalization or extension from which existing models can be derived. To show this, we can substitute particular forms of the waiting time distribution $\Psi_{T}$ within the CTRW model (Eq. 5), and see which bioturbation models are obtained. This analysis will also show how the constraints of an 'infinite signal propagation speed' and an 'infinite mixing frequency' are embedded within bioturbation models.

\section{Poisson processes}

A first case of interest is a so-called Poisson process, which applies to sequences of events that are 'randomly spaced in time'. Poisson processes have been used to describe the disintegration of radionuclides, incoming telephone calls, or chromosome break-up under irradiation (Feller 1968). The central assumption is that there is no influence of the past events on the present functioning. A Poisson process is thus 'memoryless': the fact that a particle has been displaced, does not affect its chances of being displaced again. In statistical terms, this means that the process becomes a time-homogeneous Markov process (Feller 1968). To investigate the relevance of the Poisson process for bioturbation, we focus on a single sediment particle, start the clock at time 0 , and count the number of displacements $N(t)$ within a given time period $t$. The counter $N(t)$ will increase by 1 for every bioturbation event. Bioturbation will be a Poisson process when the following condition is satisfied: the number of bioturbation events occurring in 2 intervals of the same length must be statistically independent, i.e. the probability of having $N(t)$ bioturbation events must be the same for all intervals of length $t$, independently from when we actually start the clock. If bioturbation acts as 
a Poisson process, the probability that a particle will exactly experience $n$ bioturbation events in the time interval $(0, t)$ is given by the Poisson distribution (Feller 1968, p. 447, Hughes 1995, p. 248):

$$
\operatorname{Pr}\{N(t)=n\}=\frac{\left(t / \tau_{c}\right)^{n}}{n !} \exp \left(-t / \tau_{c}\right)
$$

where $\tau_{c}$ represents the expected time interval between 'events'. The average number of particle displacements within a given time period $t$ is indeed given by $\langle N(t)\rangle=\mathrm{t} / \tau_{c}$. From Eq. (23), one can directly derive the waiting time distribution associated with a Poisson process. Upon substitution of $n=0$ in Eq. (23), one finds the probability that a particle rests for a period of at least length $t$, which is given by:

$$
\operatorname{Pr}\{N(t)=0\}=1-\int_{0}^{t} \Psi_{T}(\tau) \mathrm{d} \tau=\exp \left(-t / \tau_{c}\right)
$$

This expression features the integral of the waiting time distribution $\Psi_{T}(\tau)$. Upon differentiation with respect to time, we find that the waiting time between successive events follows the exponential distribution (Hughes 1995):

$$
\Psi_{T}(\tau)=\frac{1}{\tau_{c}} \exp \left(-\tau / \tau_{c}\right)
$$

where $\tau_{c}$ is the average waiting time. Therefore, a CTRW model with an exponential waiting time distribution is typically referred to as a Poisson process. If we insert the exponential waiting time distribution (Eq. 25) into the general CTRW equation (Eq. 5), upon differentiation with respect to time, we obtain the following integro-differential equation (see Othmer et al. 1988 for details of this derivation):

$$
\tau_{C} \frac{\partial C}{\partial t}=-C(x, t)+\int_{-\infty}^{\infty} \Psi_{L}(\lambda) C(x-\lambda, t) \mathrm{d} \lambda
$$

Eq. (26) is identical to the governing equation of the classical non-local exchange model of bioturbation originally proposed by Boudreau \& Imboden (1987). Indeed, if we introduce $x^{\prime}=x-\lambda$ and define the exchange function as:

$$
K\left(x^{\prime}, x\right)=\frac{1}{\tau_{c}} \Psi_{L}(\lambda)=\frac{1}{\tau_{c}} \Psi_{L}\left(x-x^{\prime}\right)
$$

then Eq. (26) can be readily re-arranged to the familiar non-local exchange model (Boudreau \& Imboden 1987 , Boudreau 1997):

$$
\frac{\partial C}{\partial t}=\int_{-\infty}^{\infty} K\left(x^{\prime}, x\right) C\left(x^{\prime}, t\right) \mathrm{d} x^{\prime}-\int_{-\infty}^{\infty} K\left(x, x^{\prime}\right) C(x, t) \mathrm{d} x^{\prime}
$$

In other words, a central assumption behind the classical non-local exchange model is that the mechanism of bioturbation is a Poisson process.

Note that the left-hand side of Eq. (26) (or equally Eq. 28) contains no higher temporal derivatives of the concentration, apart from the first order one. Strik- ingly, this is also the case for the diffusion equation (Eq. 21). This absence of such higher order temporal derivatives exemplifies the Markov character of the model. The Poisson process with its exponential waiting time PDF constitutes a Markovian model, in the sense that past bioturbation events do not influence the present probability of jumping. For arbitrary small time intervals, the exponential waiting distribution retains a finite probability of jumping, i.e. $\Psi_{T}(t) \rightarrow 1$ for $\tau \rightarrow 0$. Theoretically, this allows for bioturbation events to occur infinitely rapidly one after another, and, hence, there is the finite probability of finding a particle at infinite distances. This property is referred to as the infinite speed of signal propagation. The diffusion equation (Eq. 21) also shows this infinite propagation speed, and because of this it has been criticized as an unrealistic model for bioturbation (Kirwan \& Kump 1987, Boudreau 1989).

\section{Non-Markovian processes: the telegraph equation and beyond}

Physically, any natural process-hence, also a bioturbation event-requires a finite amount of time to complete. So infinitely small waiting times are unrealistic, and hence one requires that the probability of small waiting times tends to zero, i.e. $\Psi_{T}(\tau) \rightarrow 0$ for $\tau \rightarrow 0$. As noted above, the exponential distribution (Eq. 25) violates this constraint, and, because of this, it shows an infinite speed of signal propagation. However, the constraint is satisfied when the waiting time distribution $\Psi_{T}$ follows the Gamma distribution with parameter $\alpha \geq 1$ :

$$
\Psi_{T}(\tau)=\frac{1}{\tau_{c}} \frac{\alpha^{\alpha}}{\Gamma(\alpha)}\left(\frac{\tau}{\tau_{c}}\right)^{\alpha-1} \exp \left(-\alpha \tau / \tau_{c}\right)
$$

where $\Gamma(\alpha)=\int_{0}^{\infty} s^{\alpha-1} \exp (-s) \mathrm{d} s$ is the gamma function (Abramowitz \& Stegun 1964). Each curve has been 'standardized', so that it has the same averaged waiting time $\tau_{c}$. All curves with $\alpha>1$ have $\Psi_{T}(0)=0$, and so they correspond to a finite speed of signal propagation. When $\alpha=2$ is substituted into Eq. (29), we obtain the 'telegraph' waiting time distribution:

$$
\Psi_{T}(\tau)=4 \tau_{c}{ }^{-2} \tau \exp \left(-2 \tau / \tau_{c}\right)
$$

where the mean waiting time is again $\tau_{c}$. Fig. 3 illustrates the profiles for $\alpha=1$ (exponential Eq. 25), and $\alpha=$ 2 (telegraph Eq. 30). The implementation of Eq. (30) in Eq. (5) leads to:

$$
\begin{aligned}
C(x, t)= & \left(2 t / \tau_{c}+1\right) \exp \left(-2 t / \tau_{c}\right) C_{0}(x) \\
& +\left(2 / \tau_{c}\right)^{2} \int_{0}^{t} \tau \exp \left(-2 \tau / \tau_{c}\right) \int_{-\infty}^{\infty} \Psi_{L}(\lambda) C(x-\lambda, t-\tau) \mathrm{d} \lambda \mathrm{d} \tau
\end{aligned}
$$


Similar to the above, we can re-arrange Eq. (31) into a corresponding integro-differential form. After 2 consecutive differentiations with respect to time, and some rearrangements, the analogue of Eq. (26) thus becomes (Othmer et al. 1988):

$\left(\frac{\tau_{C}}{2}\right)^{2} \frac{\partial^{2} C}{\partial t^{2}}+\tau_{C} \frac{\partial C}{\partial t}=-C(x, t)+\int_{-\infty}^{\infty} \Psi_{L}(\lambda) C(x-\lambda, t) \mathrm{d} \lambda$

Eq. (32) bears a strong resemblance with the telegraph equation (Eq. 33), which was proposed by Boudreau (1989) as an alternative to the biodiffusion model, in order to cope with the problem of the infinite propagation speed:

$$
\left(\frac{\tau_{c}}{2}\right)^{2} \frac{\partial^{2} C}{\partial t^{2}}+\tau_{c} \frac{\partial C}{\partial t}=\frac{\sigma^{2}}{2} \frac{\partial^{2} C}{\partial x^{2}}
$$

Note the striking analogy between the diffusion equation (Eq. 21) and the telegraph equation (Eq. 33), on the one hand, and the 'exponential' CTRW equation (Eq. 26) and its 'telegraph extension' (Eq. 32), on the other hand.

\section{MODEL APPLICATION}

\section{Simulation of pulse-tracer addition experiments}

In a final step, we present simulations that illustrate the relation between the diffusion model (Eq. 21), the telegraph model (Eq. 33) and the general CTRW model (Eq. 5). The aim is to compare how these models simulate bioturbation over 'short' time scales. It is important to point out that 'short' is relative to the mean time interval between 2 consecutive displacements of particles (i.e. mean waiting time $\tau_{c}$ ). Different benthic communities will induce particle displacement characterized by a different mean waiting time $\tau_{c}$ and consequently they will have a different characteristic time

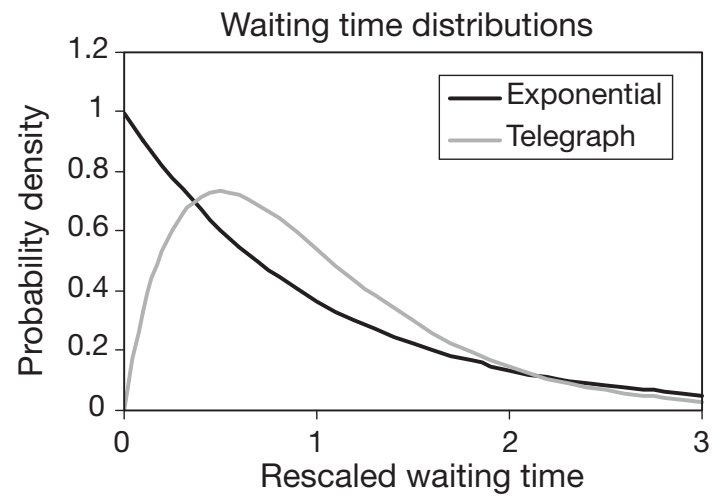

scale of mixing. Deep-sea environments are less intensely mixed than coastal environments, and hence the mean waiting time $\tau_{c}$ associated with bioturbation in benthic communities of the deep sea can be far greater than that of coastal communities. Accordingly, the notion 'short time scales' will have a different meaning in different sediment environments.

A widely implemented technique to study bioturbation over short time scales is via a tracer pulse addition experiment (e.g. Gerino 1990, Fornes et al. 1999, 2001, Solan et al. 2004, Fernandes et al. 2006, Duport et al. 2007). A layer of tracer particles is added to the SWI, and its subsequent down-mixing into the sediment is followed. Here, we simulate such a tracer pulse addition for inert particles (e.g. fluorescent luminophores), assuming that the sediment is initially covered by a tracer layer of $5 \mathrm{~mm}$ thickness. The tracer concentration is defined as equal to 1 within this initial layer. The 'short time scale' over which the tracer mixing is simulated covers 10 times the mean waiting time $\tau_{c}$.

\section{Four different bioturbation regimes}

We simulated the same pulse-tracer experiment with 6 different models: the biodiffusion model (Eq. 21), the telegraph model (Eq. 33) and 4 different versions of the CTRW model (Eq. 5), which represent 4 different bioturbation regimes. A bioturbation regime refers to the particle spreading process within a particular sediment setting. This process will depend on the type and number of organisms that are present, and on the intensity and the mechanism of their activity (e.g. deposit-feeding, burrowing). From the perspective of the CTRW model, a given bioturbation regime is completely characterized by its bioturbation fingerprint, i.e. a particular combination of a waiting time distribution $\Psi_{T}$ and a jump length distribution $\Psi_{L}$.

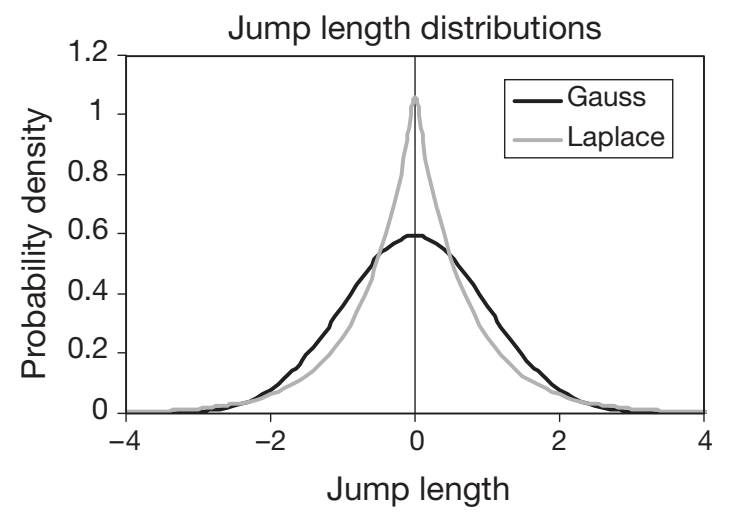

Fig. 3. Four different bioturbation fingerprints are created by the combination of 2 waiting time distributions (left panel) and 2 jump length distributions (right panel). Exponential and telegraph distributions are used for the waiting time probability distribution function (PDF, left panel). Laplacian and Gaussian distributions are used for the jump length PDF (right panel). See 'Model application; Four different bioturbation regimes' for the mathematical formulae that describe these distributions 
Once these distributions are known, the CTRW model formulation is complete. Here, 4 different CTRW models were made by combining 2 waiting time distributions $\Psi_{T}$ and 2 jump length distributions $\Psi_{L}$. For the waiting time distribution, we either used the Markovian exponential distribution (Eq. 25) ( $\alpha=1$ in Eq. 29) or the non-Markovian Gamma distribution (Eq. 30) ( $\alpha=2$ in Eq. 29). To construct the jump length distributions $\Psi_{L}$, we used the following family of curves:

$\Psi_{L}(\lambda)=\frac{1}{2 \sigma \Gamma(1+1 / m)} \sqrt{\frac{\Gamma(1 / m)}{\Gamma(3 / m)}} \exp \left[-\sqrt{\frac{\Gamma(1 / m)}{\Gamma(3 / m)}}\left(\frac{\lambda}{\sigma}\right)^{m}\right]$

where $\Gamma(x)$ again denotes the Gamma function. The curves (Eq. 34) have a peak at $\lambda=0$, and the same standard deviation $\sigma^{2}$, and zero skewness. The curves have, however, different kurtosis, depending on the value of $m$. The values $m=1$ and $m=2$ are used here (Fig. 3). When $m=1$, Eq. (34) becomes the Laplace distribution:

$$
\Psi_{L}(\lambda)=\frac{1}{\sigma \sqrt{2}} \exp \left(-\frac{\sqrt{2}|\lambda|}{\sigma}\right)
$$

When $m=2$, Eq. (34) becomes the familiar Gaussian distribution:

$$
\Psi_{L}(\lambda)=\frac{1}{\sigma \sqrt{2 \pi}} \exp \left(-\frac{\lambda^{2}}{2 \sigma^{2}}\right)
$$

Combining the 2 waiting time distributions (Eqs. 25 \& 30) with the 2 step length distributions (Eqs. 35 \& 36) gives rise to 4 different bioturbation fingerprints (Markovian $\times$ Gaussian; Markovian $\times$ Laplacian; nonMarkovian $\times$ Gaussian; non-Markovian $\times$ Laplacian) . Note that all 4 CTRW models, as well as the biodiffusion equation (Eq. 21) and the telegraph equation (Eq. 33), feature the same 2 parameters $\sigma$ and $\tau_{c}$. These parameters are given the same values in all 6 simulations.

\section{Simulation results}

The 6 model simulations of the same pulse-tracer addition experiment are plotted in Fig. 4. The longtime behaviour of the models is striking: at about 10 times the average waiting time, all 6 solutions coincide. This is exactly what random-walk theory predicts (Hughes 1995, Metzler \& Klafter 2000). All models were designed to have the same mixing intensity, as specified by the biodiffusion coefficient (Eq. 22). To this end, the distributions were standardized, so that the mean waiting time $\tau_{c}$ and step length variance $\sigma^{2}$ were the same for all 4 CTRW bioturbation regimes. The same parameter values for $\tau_{c}$ and $\sigma$ were used in the diffusion equation (Eq. 21) and the telegraph equation (Eq. 33). The merging of the simulated profiles at long times also shows that our numerical solution scheme for the CTRW model is accurate.

At short times, there is a marked difference between the 4 solutions of the CTRW model on the one hand, and the solutions of the biodiffusion and telegraph models on the other hand. The CTRW profiles all consist of 2 parts: (1) a 'blocky' zone corresponding to the initial tracer layer and (2) a 'smooth' zone beneath the initial layer, which shows a gradually decreasing tracer concentration with depth. The 'blocky' zone contains particles that have not yet been moved. Over time, this zone is gradually eroded, as more and more particles are mixed down. The 'smooth' underlying zone contains particles that have been displaced. Neither the diffusion model, nor the telegraph model, shows this characteristic 2-zone pattern. Instead they give rise to a single, more or less homogeneously reworked zone near the sediment surface. As a result, both diffusion and telegraph models predict considerably lower concentrations at the sediment surface during the initial stages of the experiment, when compared to the 4 CTRW models. When comparing the profiles of the 4 CTRW models, the differences are minor. For the same waiting time distribution, the profiles corresponding to the Laplacian and Gaussian step length distributions nearly coincide. For the same step length distribution, the profile corresponding to the Gamma waiting time distribution (Eq. 30) shows slower erosion of the initial tracer layer than the profile obtained from the exponential distribution (Eq. 25).

Finally, there are also 2 marked differences between the tracer profiles generated by the biodiffusion and telegraph models. The concentration profile produced by the telegraph equation at short times takes on an unusual, irregular shape: it shows a marked 'truncation', which abruptly limits the penetration of particles. Just before this truncation, the profiles exhibit a strange accumulation of tracer, which appears like a 'chimney'. Effectively, the chimney appears to sit on the top of a truncated diffusion profile (compare the profiles at $t=0.5$; the first centimetre of the diffusion profile is very similar to that of the telegraph profile). Okubo (1971) has investigated the mathematical details of the solution of the telegraph equation, and explained the origin of these 2 peculiar features. The solution of the telegraph model actually consists of 2 parts: a wave-like and a diffusive-like component (respectively corresponding to the second-order and first-order temporal derivative in Eq. 33). The truncation then corresponds to the diffusive-like component, where particles can only move downwards with a finite propagation speed $c=\sqrt{2} \sigma / \tau_{c}$. Accordingly, there are no particles beyond $x=c t$, which is the depth where the tracer profile is truncated. Conversely, the chimney at the front edge is due to the wave-like com- 
Diffusive (infinite signal speed)


Fig. 4. Transient simulations of the same pulse-tracer addition experiment by 6 different models. The initial tracer layer at the surface is given by the dotted line. Left and right panels each show the output of 3 models at 4 different times. Left panels: thin continuous line: continuous-time random-walk (CTRW) model with exponential waiting time and Laplacian jump length; thick continuous line: CTRW model with exponential waiting time and Gaussian jump length; dashed line: asymptotic biodiffusion model. Right panels: thin continuous line: CTRW model with 'telegraph' waiting time and Laplacian jump length; thick continuous line: CTRW model with 'telegraph' waiting time and Gaussian jump length; dashed line: solution of the associated telegraph equation. In all simulations we used the same characteristic length scale $\sigma=2 \mathrm{~cm}$. The time elapsed is expressed relative to the mean waiting time between particle displacements (e.g. $t=10$ denotes a simulation period of 10 times the mean waiting time)

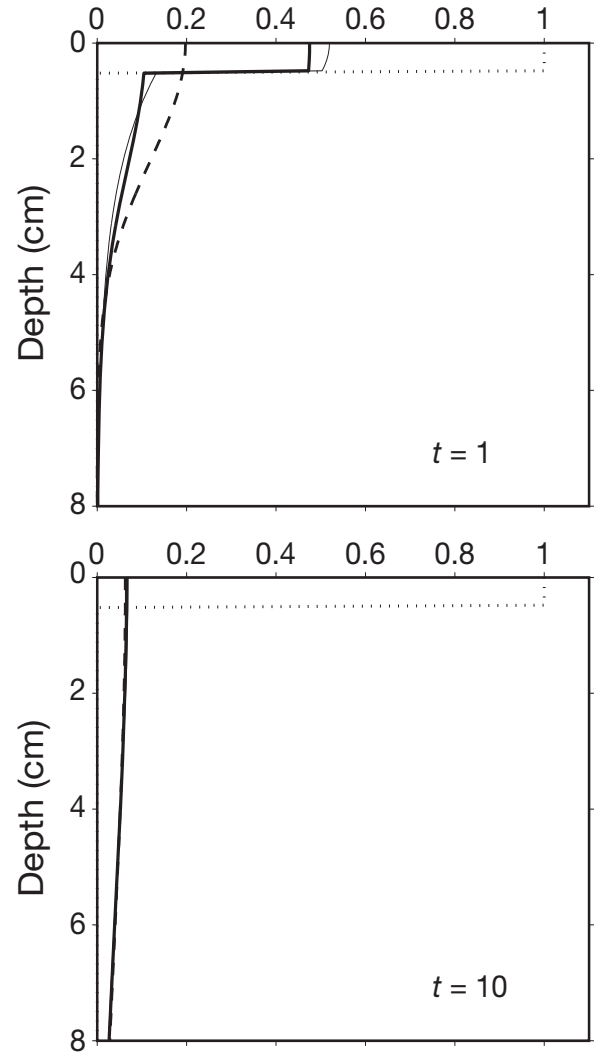

Telegraph (finite signal speed)
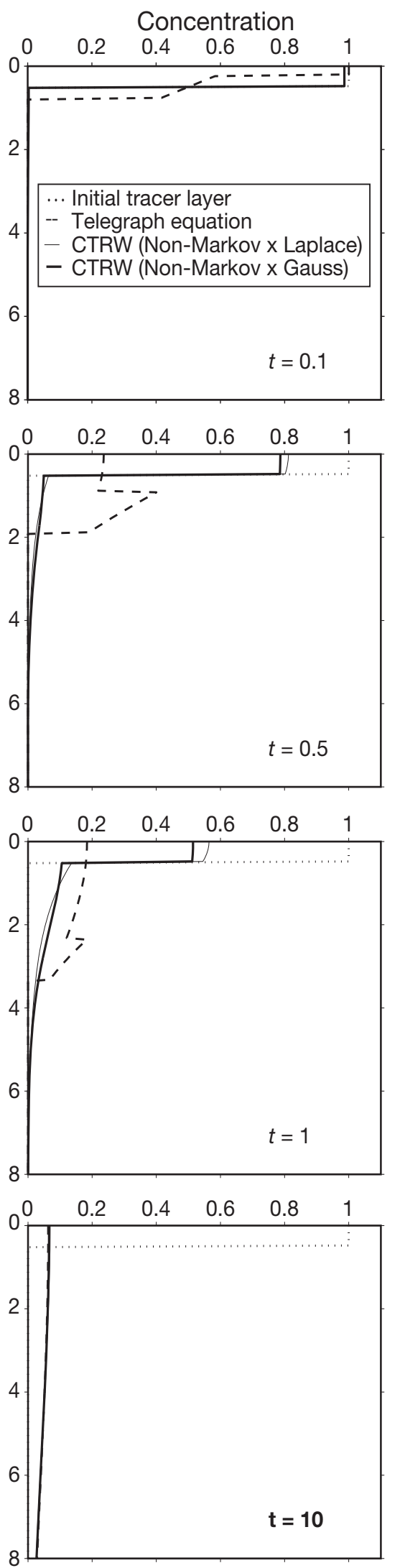
ponent, and represents a damped wave motion of the initial conditions. The decay of the chimney is on the order of $\sim \tau_{c} / 4$, and therefore the chimney has virtually disappeared when $t>\tau_{c}$.

\section{DISCUSSION}

In bioturbation research, pulse-tracer additions form a popular experimental method. In these methods, a pulse of inert tracer particles is deposited at the sediment surface, and the evolution of the vertical tracer profile is followed over time. These pulse experiments last only a short period compared to the intrinsic mixing time of the environment, typically on the order of hours (e.g. 48 h; Solan et al. 2004) to days (e.g. $22 \mathrm{~d}$ in Gerino 1990; 28 d in Fernandes et al. 2006) in nearshore conditions, and years in the deep sea (e.g. Wheatcroft 1991). These observational times should be compared to the inherent time scale of bioturbation, i.e. the mean waiting time $\tau_{c}$ between consecutive displacements of a given particle. At present, we have no accurate estimates of the mean waiting time $\tau_{c}$ between bioturbation events, since we do not have experimental techniques that can track the movement of individual particles. Based on population reworking rates of deposit feeders, Wheatcroft et al. (1990) and Meysman et al. (2003) crudely estimated that $\tau_{c}$ must range on the order of hours to months for natural communities. Recently, Maire et al. (2007) fitted the Markov $\times$ Gaussian CTRW to high-resolution luminophore data obtained in microcosm experiments. They found that $\tau_{c}$ ranged between 1 to $10 \mathrm{~h}$ in the summertime $\left(D_{\mathrm{b}} \sim 40 \mathrm{~cm}^{2} \mathrm{yr}^{-1}\right)$ and 10 to $30 \mathrm{~h}$ in the wintertime $\left(D_{\mathrm{b}} \sim 5 \mathrm{~cm}^{2} \mathrm{yr}^{-1}\right)$ for sediments mixed by the burrowing bivalve Abra ovata. In these microcosm experiments, the observational time scale was $48 \mathrm{~h}$. As a result, the observational time scale of the experiment and the intrinsic process time scale of bioturbation are of the same order. In other words, the particles that are observed in pulse-tracer experiments will only experience a limited number of bioturbation events.

Until now, this aspect of short observational time scales has received very little consideration, when interpreting the results of pulse-tracer experiments. In the standard procedure, the biodiffusion model (Eq. 21) is applied, and a biodiffusion coefficient $D_{\mathrm{b}}$ is derived by suitably fitting to the tracer data profiles (with some exceptions: see Shull \& Yasuda 2001 and Solan et al. 2004 for the application of non-local models to tracer data). However, it usually is not investigated whether the assumptions underlying the biodiffusion model are valid at such short time scales. Note that the question is not whether the biodiffusion model can be applied to tracer profiles at short time scales (this can always be done), but whether the resulting values for the biodiffusion coefficient are meaningful. Recently, Reed et al. (2006) showed that strong artefacts can arise when the biodiffusion model is applied to steady-state profiles of short-lived radiotracers. Based on lattice-automaton simulations (i.e. synthetic data generated from a virtual sediment), they showed that tracer-derived biodiffusion coefficients, obtained by fitting tracer profiles of short-lived radionuclides, may strongly deviate (by orders of magnitude) from biodiffusion coefficients derived from particle tracking, which represent the true mixing intensity of the sediment (i.e. the $D_{\mathrm{b}}$ obtained from Eq. 22). Our analysis of transient pulse-tracer experiments provides an explanation for these problems with the biodiffusion model at short time scales: the infinite speed of signal propagation and the assumption of infinitely short waiting times.

\section{Infinite signal propagation speed: the telegraph equation as an alternative to the biodiffusion model}

One problem with the biodiffusion model is the infinite speed of signal propagation. Shortly after the start of the pulse-tracer experiment, the biodiffusion model will predict that tracer particles penetrate too far down into the sediment (see Fig. 4, left panel $t=0.1$ ). To correct this artefact, Boudreau (1989) suggested the telegraph equation (Eq. 33) as an alternative, which has indeed a finite velocity of signal propagation (Monin 1959, Okubo 1971). However, until the present time, no one has actually used the telegraph equation to simulate or analyse a pulse-tracer addition experiment. This is done here, and the results are quite disconcerting for the telegraph model. As shown in Fig. 4, the concentration profile produced by the telegraph equation includes 2 strange and unrealistic features: a truncation of the tracer profile and a strange accumulation of tracer near the truncation depth. In his detailed mathematical investigation of the telegraph equation, Okubo (1971, p. 28) referred to the truncation of the tracer profile as an 'advancing front', while the accumulation was termed a 'heaping of substance near the front edge'. Okubo (1971) noted that the presence of 'an advancing front where substance is heaped' does not match observations in experiments on oceanic diffusion. Nonetheless, Okubo (1971) attributed the 'heaping effect' to unrealistic (Dirac pulse) initial conditions, and he (still) concluded that the telegraph equation could produce realistic tracer patterns. We think that this interpretation of Okubo (1971) is far too merciful for the telegraph equation as a tracer mixing model. In bioturbation studies, neither sharp truncations, nor moving chimneys have been observed in 
actual pulse-tracer experiments (see e.g. Fig. 4 in Maire et al. 2007). Unlike Okubo's (1971) assertion, the accumulation of tracer at the front edge does not result from unrealistic initial conditions, but is a fundamental aspect of the telegraph's solution. Given an initial layer of tracer at the sediment surface, the strange accumulation at depth will always be prominently present in the telegraph tracer profile, because a wave-like subcomponent is always present within its solution. In conclusion, the replacement of the diffusion model by the telegraph equation as a bioturbation model solves one minor problem (the infinite propagation speed), but, at the same time, it introduces a far more important bias (the introduction of unrealistic truncation and accumulation features in tracer profiles). Accordingly, the telegraph equation does not form a suitable alternative to the biodiffusion model at short time scales.

\section{The problem of infinitely short waiting times}

One can ask whether the infinite speed of signal propagation is the true critical issue when modelling particle mixing at short time scales. Our simulations indicate that it is not. To this end, we compared a CTRW model with an infinite signal propagation speed (exponential waiting time distribution; left column of Fig. 4) and the same model with a finite signal propagation speed (Gamma waiting time distribution; right column of Fig. 4). The differences between the resulting CTRW tracer profiles are very small, and would hardly be distinguishable in the presence of actual data, given the typical error bars associated with such data. In contrast, the crucial dichotomy in Fig. 4 occurs between the differential equation models on the one hand (the diffusion and telegraph solution), and the integral equation models on the other hand (the 4 CTRW solutions). Both categories of models display fundamentally different model behaviour at short time scales. In the CTRW models, the initial tracer layer 'persists' in the model solutions, and only gradually fades with time. In contrast, the initial conditions are immediately wiped out in the differential equation models.

So why do the biodiffusion and telegraph models immediately clear their 'memory' of the initial tracer layer? The answer to this question provides a new insight into the assumptions and applicability of bioturbation models. The difference between integral models such as the CTRW presented here, and differential models such as the biodiffusion (Eq. 21) and telegraph (Eq. 33) models, but also the classical non-local exchange model (Eq. 28), boils down essentially to the underlying assumption about the waiting time between bioturbation events. The integral equation (Eq. 5) of the
CTRW model explicitly describes the waiting time between bioturbation events via a probability distribution, and as a consequence the mean waiting time $\tau_{c}$ has a finite value. In contrast, models that feature temporal derivatives implicitly assume an infinitely small $\tau_{c}$. In the derivations of these models, one performs a series expansion of the concentration about the time, and subsequently one performs a limit operation $\tau_{c} \rightarrow 0$. This was shown by Wheatcroft et al. (1990) for the biodiffusion model (Eq. 21), by Boudreau (1989) for the telegraph model (Eq. 33), and by Meysman et al. (2003) for the non-local exchange model (Eq. 26). In other words, temporal differentiation implies a limit operation where the mixing time scale $\tau_{c}$ becomes infinitely small. An infinitely small waiting time then implies that all particles are constantly being displaced. In other words, the biodiffusion and other differential models assume that all particles within the initial tracer layer are immediately affected by sediment reworking. This explains why the initial conditions are immediately 'erased' from the tracer profile. In contrast, in the CTRW model, particles are not displaced immediately. Some particles remain undisturbed for a finite time, and this is the reason why the initial tracer layer persists for some time in the tracer profile.

In summary, our analysis brings up a critical and previously unrecognized issue in bioturbation modelling. In a seminal paper on bioturbation modelling, Wheatcroft et al. (1990) introduced the concept of a waiting time between bioturbation events. Here, we elaborate when and why this waiting time becomes important: models should account for a finite waiting time between bioturbation events when modelling tracer behaviour at short observational time scales. The biodiffusion model implicitly assumes an infinitely small waiting time, as does the telegraph equation and any other differential model. Note that even the non-local exchange model of Boudreau \& Imboden (1987) also comprises a differential model, and so it also implicitly assumes an infinitely small waiting time. This model has been frequently heralded as a more realistic alternative to the biodiffusion model. Although not explicitly tested here, our analysis questions the usefulness of non-local exchange models at short time scales. When modelling tracers over short time scales, the waiting time between bioturbation events becomes important, and so the waiting time cannot be idealized as infinitely small.

\section{Implications for short-term tracer studies}

The discussion in the previous section about the 'persisting' initial layers and importance of finite waiting times was solely based on model analysis (the compari- 
son of the CTRW model to other models in Fig. 4). So, are there any empirical data that support these arguments? The relevance of bioturbation models with finite waiting times was recently shown in a tracer study by Maire et al. (2007). The biological reworking of the bivalve Abra ovata was followed in thin aquaria over a short time period of $48 \mathrm{~h}$. Particle dispersal was quantified in a classical pulse addition experiment, where a thin layer of luminophores was added on top of the sediment. Images were taken from the side of the aquaria, which then generated 2-dimensional (2D) tracer distributions. It was observed that the bivalve reworking was spatially patchy: some zones were intensively reworked, while, in other zones, the initial tracer layer remained unaltered. Over time, the bivalves relocated, and so gradually more and more of the sediment surface became reworked. When generating 1D tracer profiles from the 2D images, the sediment is laterally averaged, and, hence, 'mixed' and 'unmixed' zones are merged into single tracer depth profiles. This effectively resulted in 1D tracer profiles that are very similar to the CTRW solutions as presented in Fig. 4: the initial tracer layer 'persists' for some time in consecutive tracer profiles (see Fig. 4 in Maire et al. 2007). Only gradually, as the bivalves relocate, is more and more of the 'pristine' sediment (i.e. with intact tracer surface layer) reworked. Maire et al. (2007) showed that the interpretation of such profiles with the standard biodiffusion model leads to significant bias in the mixing intensity. The biodiffusion model assumes that all particles within the initial layer are immediately affected (infinitely frequent bioturbation events), and hence it cannot cope with lateral heterogeneity in bivalve reworking activity. We believe this observation is not specific to A. ovata, but applies to bioturbation in general. Over short time scales, lateral heterogeneity in bioturbation activity cannot be ignored in tracer studies. In terms of modelling, this implies that when 1D bioturbation models are applied over short time scales, one should account for differential timing in particle displacements. A tracer layer that is initially deposited at the SWI, should be gradually mixed down, rather than that all particles are displaced immediately and synchronously. The CTRW model presented here allows for such differential timing: lateral spatial heterogeneity in reworking is essentially translated into vertical stochasticity of particle displacement.

\section{CONCLUSIONS}

In recent years, new experimental techniques have been developed that generate tracer profiles of a high spatial ( 1 $\mathrm{mm})$ and high temporal resolution ( 10 min) (Gilbert et al. 2003, Solan et al. 2004, O'Reilly et al. 2006, Maire et al. 2007). Accordingly, one requires suitable tracer models that adequately describe the resulting tracer data at such short time scales. We have identified 2 issues with quantifying bioturbation over short time scales: (1) the infinitely fast propagation of tracer to finite depths and (2) the assumption that bioturbation events occur infinitely frequently. Our model analysis shows that the latter issue is far more relevant to tracer studies than the former. In short-term tracer studies, lateral spatial heterogeneity in reworking becomes important, which translates into vertical particle displacements with finite waiting times. Organisms do not rework the surface layer of sediments and soils in a homogeneous fashion. Rather, bioturbation should be regarded as a process of sediment patches that are individually reworked and that shift with time when organisms relocate. In tracer pulse addition experiments, this lateral heterogeneity in reworking results in conspicuous 1D tracer profiles, where initial conditions 'persist'.

The issue of waiting times has important repercussions for the tracer models that are used in combination with experimental tracer studies. At present, models are used that are stated in a differential form, of which the biodiffusion model (Eq. 21) is by far the most popular. We have shown that such 'differential' models implicitly assume that bioturbation events occur with infinite frequency. At short time scales, the application of such models is problematic, and may lead to biased predictions. When applied to short time scales, bioturbation models should explicitly account for a finite waiting time between bioturbation events. Here, we advance the CTRW model as a suitable description of bioturbation, which is able to cope with lateral spatial heterogeneity in reworking, However, at long time scales, the application of biodiffusion is not a problem. After a sufficient amount of time (i.e. a sufficient amount of bioturbation events), the CTRW model profile becomes identical to that of the biodiffusion model. At this stage, the more complex CTRW model loses its advantage over the much simpler biodiffusion model. So, in conclusion, it is not a good idea to use the biodiffusion model at short time scales, but there seems no reason not to use it at long time scales.

Acknowledgements. This research was supported by grants from the EU (MARBEF, 505446), the Netherlands Organization for Scientific Research (NWO PIONIER, 833.02.2002), and contributes to the Darwin Institute for Biogeology. This is Publication no. 4323 of the Netherlands Institute of Ecology (NIOO-KNAW).

\section{LITERATURE CITED}

Abramowitz M, Stegun IA (1964) Handbook of mathematical functions with formulas, graphs, and mathematical tables. Dover, New York

Aller RC (1988) Benthic fauna and biogeochemical processes 
in marine sediments: the role of burrow structures. In: Blackburn TH, Sorensen J (eds) Nitrogen cycling in coastal marine sediments. John Wiley \& Sons, New York, p 301-338

Aller RC, Yingst JY (1978) Biogeochemistry of tube-dwellings: a study of the sedentary polychaete Amphitrite ornata (Leidy). J Mar Res 36:201-254

Boudreau BP (1986) Mathematics of tracer mixing in sediments. I. Spatially dependent, diffusive mixing. Am J Sci 286:161-198

Boudreau BP (1989) The diffusion and telegraph equations in diagenetic modeling. Geochim Cosmochim Acta 53: 1857-1866

Boudreau BP (1997) Diagenetic models and their implementation. Springer, Berlin

Boudreau BP, Imboden DM (1987) Mathematics of tracer mixing in sediments. III. The theory of nonlocal mixing within sediments. Am J Sci 287:693-719

Cadée GC (2001) Sediment dynamics by bioturbating organisms. In: Reise $\mathrm{K}$ (ed) Ecological comparisons of sedimentary shores. Springer, Berlin, p 127-148

Duport E, Gilbert F, Poggiale JC, Dedieu K, Rabouille C, Stora G (2007) Benthic macrofauna and sediment reworking quantification in contrasted environments in the Thau Lagoon. Estuar Coast Shelf Sci 72:522-533

Feller W (1968) An introduction to probability theory and its applications. John Wiley \& Sons, New York

Fernandes S, Meysman FJR, Sobral P (2006) The influence of $\mathrm{Cu}$ contamination on Nereis diversicolor bioturbation. Mar Chem 102:148-158

Fornes WL, Demaster DJ, Levin LA, Blair NE (1999) Bioturbation and particle transport in Carolina slope sediments: a radiochemical approach. J Mar Res 57:335-355

Fornes WL, Demaster DJ, Smith CR (2001) A particle introduction experiment in Santa Catalina basin sediments: testing the age-dependent mixing hypothesis. J Mar Res 59:97-112

Gardiner CW (2002) Handbook of stochastic methods for physics, chemistry and the natural sciences. SpringerVerlag, Berlin

Gerino M (1990) The effects of bioturbation on particle redistribution in Mediterranean coastal sediment - preliminary results. Hydrobiologia 207:251-258

Gilbert F, Hulth S, Stromberg N, Ringdahl K, Poggiale JC (2003) 2-D optical quantification of particle reworking activities in marine surface sediments. J Exp Mar Biol Ecol 285-286:251-263

Goldberg ED, Koide M (1962) Geochronological studies of deep-sea sediments by the Io/Th method. Geochim Cosmochim Acta 26:417-450

Guinasso NL, Schink DR (1975) Quantitative estimates of biological mixing rates in abyssal sediments. J Geophys Res 80:3032-3043

Hughes BD (1995) Random walks and random environments, Vol 1. Random walks. Clarendon Press, Oxford

Kirwan AD, Kump LR (1987) Models of geochemical systems for mixture theory: diffusion. Geochim Cosmochim Acta 51:1219-1226

Mahaut ML, Graf G (1987) A luminophore tracer technique for bioturbation studies. Oceanol Acta 10:323-328

- Maire O, Duchene JC, Gremare A, Malyuga VS, Meysman FJR (2007) A comparison of sediment reworking rates by the surface deposit-feeding bivalve Abra ovata during summertime and wintertime, with a comparison between two models of sediment reworking. J Exp Mar Biol Ecol 343:21-36

Metzler R, Klafter J (2000) The random walk's guide to anomalous diffusion: a fractional dynamics approach. Phys Rep 339:1-77

Meysman FJR, Boudreau BP, Middelburg JJ (2003) Relations between local, nonlocal, discrete and continuous models of bioturbation. J Mar Res 61:391-410

Meysman FJR, Middelburg JJ, Heip CHR (2006) Bioturbation: a fresh look at Darwin's last idea. Trends Ecol Evol 21:688-695

Meysman FJR, Malyuga VS, Boudreau BP, Middelburg JJ (2008) A generalized stochastic approach to particle dispersal in soils and sediments. Geochim Cosmochim Acta (in press)

Monin AS (1959) Smoke propagation in the surface layer of the atmosphere. Adv Geophys 6:331-343

> Montroll EW, Weiss GH (1965) Random walks on lattices. II. J Math Phys 6:167-181

Okubo A (1971) Application of the telegraph equation to oceanic diffusion: another mathematical model. Tech Rep 69, Chesapeake Bay Institute, John Hopkins University, Baltimore

O'Reilly R, Kennedy R, Patterson A (2006) Destruction of conspecific bioturbation structures by Amphiura filiformis (Ophiuroida): evidence from luminophore tracers and in situ time-lapse sediment-profile imagery. Mar Ecol Prog Ser 315:99-111

Othmer HG, Dunbar SR, Alt W (1988) Models of dispersal in biological systems. J Math Biol 26:263-298

Piessens R, de Doncker-Kapenga E, Überhuber C, Kahaner D (1983) QUADPACK: a subroutine package for automatic integration. Springer-Verlag, Berlin

Reed DC, Huang K, Boudreau BP, Meysman FJR (2006) Steady-state tracer dynamics in a lattice-automaton model of bioturbation. Geochim Cosmochim Acta 70:5855-5867

Rhoads DC (1974) Organism-sediment relations on the muddy sea floor. Oceanogr Mar Biol 12:263-300

Richter R (1952) Fluidal-Textur in Sediment-Gesteinen und über Sedifluktion überhaupt. Notizbl Hess Landesamtes Bodenforsch Wiesb 3:67-81

Shull DH, Yasuda M (2001) Size-selective downward particle transport by cirratulid polychaetes. J Mar Res 59:453-473

Solan M, Wigham BD (2005) Biogenic particle reworking and bacterial-invertebrate interactions in marine sediments. In: Kristensen E, Haese RR, Kostka JE (eds) Interactions between macro- and microorganisms in marine sediments. Coastal Estuar Stud 60:105-124

Solan M, Wigham BD, Hudson IR, Kennedy R and others (2004) In situ quantification of bioturbation using timelapse fluorescent sediment profile imaging (f-SPI), luminophore tracers and model simulation. Mar Ecol Prog Ser 271:1-12

Wheatcroft RA (1991) Conservative tracer study of horizontal sediment mixing rates in a bathyal basin, California borderland. J Mar Res 49:565-588

Wheatcroft RA, Jumars PA, Smith CR, Nowell ARM (1990) A mechanistic view of the particulate biodiffusion coefficient-step lengths, rest periods and transport directions. J Mar Res 48:177-207 
Appendix 1. Reformulation of Eq. (18) in terms of real integrals

Eq. (18) is not solved as such, but is first reformulated before numerical integration. Taking into account that the Fourier transform $\hat{\Psi}_{L}(k)$ vanishes as $k$ increases, we can improve the convergence of the integral on the right-hand side as follows:

$$
\begin{aligned}
C(x, t)= & G(t) C_{0}(x) \\
& +\frac{1}{4 \pi^{2} i} \int_{-\infty}^{\infty} \exp (-i k x) \int_{\gamma-i \infty}^{\gamma+i \infty} \frac{1-\tilde{\Psi}_{T}(s)}{s}\left[\frac{1}{1-\tilde{\Psi}_{T}(s) \hat{\Psi}_{L}(k)}-1\right] \\
& \exp (s t) \mathrm{d} s \mathrm{~d} k
\end{aligned}
$$

Here, the following auxiliary function $G(t)$ is introduced as:

$$
G(t)=1-\frac{1}{2 \pi i} \int_{\sigma-i \infty}^{\sigma+i \infty} \frac{\tilde{\Psi}_{T}(s)}{s} \exp (s t) \mathrm{d} s
$$

The solution of our initial value problem then requires the evaluation of the integrals in Eqs. (A1) \& (A2). The contour integrals in Eq. (A1) can be readily represented in terms of real integrals. For this purpose, the variable of integration in the second integral can be rewritten as $s=$ $\gamma+i \xi$. Because the waiting time distribution $\Psi_{L}$ is an even function, it is evident that its Fourier transform $\hat{\Psi}_{L}(k)$ is also an even function, which takes real values on the straight line $-\infty<k<\infty$. It is easy to see that the imaginary part of the integrand in Eq. (A1) is an odd function of $\xi$, and therefore vanishes upon integration. Thus, in order to evaluate the second integral in Eq. (A1), one needs only to integrate the real part of the integrand. In a similar manner, the integral with respect to $k$ requires only the integration of the real part of the integrand. As a result, Eq. (A1) takes the final form:

$$
\begin{aligned}
C(x, t)= & G(t) C_{0}(x) \\
& +\frac{\exp (\gamma t)}{4 \pi^{2}} \int_{-\infty}^{\infty} \hat{C}_{0}(k) \cos (k x) \int_{-\infty}^{\infty} H(s, k) \mathrm{d} \xi \mathrm{d} k
\end{aligned}
$$

where $s=\gamma+i \xi$. The auxiliary functions $G$ and $H$ are, respectively:

$$
\begin{aligned}
& G(t)= 1-\frac{\exp (\gamma t)}{\pi} \\
& \int_{0}^{\infty}\left[\operatorname{Re}\left(\frac{\tilde{\phi}(s)}{s}\right) \cos (\xi t)-\operatorname{Im}\left(\frac{\tilde{\phi}(s)}{s}\right) \sin (\xi t)\right] \mathrm{d} \xi \\
& H(s, k)=\operatorname{Re}\left[\frac{1-\tilde{\Psi}_{T}(s)}{s}\left(\frac{1}{1-\tilde{\Psi}_{T}(s) \hat{\Psi}_{L}(k)}-1\right) \exp (i \gamma t)\right]
\end{aligned}
$$

The representation of Eqs. (A3) to (A5) represents the form that was actually used in all numerical simulations. 